UvA-DARE (Digital Academic Repository)

\title{
Hard burst emission from the soft gamma repeater SGR 1900+14
}

Woods, P.M.; Kouveliotou, C.; van Paradijs, J.; Briggs, M.S.; Hurley, K.; Göğüș, E.; Preece, R.D.; Giblin, T.W.; Thompson, C.; Duncan, R.C.

Published in:

Astrophysical Journal Letters

DOI:

$10.1086 / 312399$

Link to publication

Citation for published version (APA):

Woods, P. M., Kouveliotou, C., van Paradijs, J., Briggs, M. S., Hurley, K., Göğüș, E., Preece, R. D., Giblin, T. W., Thompson, C., \& Duncan, R. C. (1999). Hard burst emission from the soft gamma repeater SGR $1900+14$. Astrophysical Journal Letters, 527(1), L47-L50. https://doi.org/10.1086/312399

\section{General rights}

It is not permitted to download or to forward/distribute the text or part of it without the consent of the author(s) and/or copyright holder(s), other than for strictly personal, individual use, unless the work is under an open content license (like Creative Commons).

Disclaimer/Complaints regulations

If you believe that digital publication of certain material infringes any of your rights or (privacy) interests, please let the Library know, stating your reasons. In case of a legitimate complaint, the Library will make the material inaccessible and/or remove it from the website. Please Ask the Library: https://uba.uva.nl/en/contact, or a letter to: Library of the University of Amsterdam, Secretariat, Singel 425, 1012 WP Amsterdam, The Netherlands. You will be contacted as soon as possible. 


\title{
HARD BURST EMISSION FROM THE SOFT GAMMA REPEATER SGR 1900+14
}

\author{
Peter M. Woods, ${ }^{1,2}$ Chryssa Kouveliotou, ${ }^{2,3}$ Jan van Paradius, ${ }^{1,4}$ Michael S. Briggs, ${ }^{1,2}$ \\ Kevin Hurley, ${ }^{5}$ Ersin GöĞüşs, ${ }^{1,2}$ Robert D. Preece, ${ }^{1,2}$ Timothy W. Giblin, ${ }^{1,2}$ \\ Christopher Thompson, ${ }^{6}$ and Robert C. Duncan ${ }^{7}$ \\ Received 1999 September 15; accepted 1999 October 12; published 1999 November 9
}

\begin{abstract}
We present evidence for burst emission from SGR 1900+14 with a power-law high-energy spectrum extending beyond $500 \mathrm{keV}$. Unlike previous detections of high-energy photons during bursts from soft gamma repeaters (SGRs), these emissions are not associated with extraordinarily bright flares. Not only is the emission hard, but the spectra are better fitted by D. Band's gamma-ray burst (GRB) function rather than by the traditional optically thin thermal bremsstrahlung model. We find that the spectral evolution within these hard events obeys a hardness/ intensity anticorrelation. Temporally, these events are distinct from typical SGR burst emissions in that they are longer $(\sim 1 \mathrm{~s})$ and have relatively smooth profiles. Despite a difference in peak luminosity of $\gtrsim 10^{11}$ between these bursts from SGR $1900+14$ and cosmological GRBs, there are striking temporal and spectral similarities between the two kinds of bursts, aside from spectral evolution. We outline an interpretation of these events in the context of the magnetar model.
\end{abstract}

Subject headings: pulsars: general — stars: individual (SGR 1900+14) - X-rays: bursts

\section{INTRODUCTION}

Soft gamma repeaters (SGRs) constitute a group of highenergy transients named for the observed characteristics that set them apart from classical gamma-ray bursts (GRBs). SGRs emit brief ( $\sim 0.1 \mathrm{~s})$, intense (up to $10^{3}-10^{4} L_{\mathrm{Edd}}$ ) bursts of lowenergy gamma-rays with recurrence times that range from seconds to years (Kouveliotou 1995). The vast majority of SGR burst spectra ( $\gtrsim 20 \mathrm{keV}$ ) can be fitted mathematically by an optically thin thermal bremsstrahlung (OTTB) model with temperatures between 20 and $35 \mathrm{keV}$ (Fenimore, Laros, \& Ulmer 1994; Göğüs et al. 1999); however, there is no physical justification for this model (Fenimore et al. 1994; Thompson \& Duncan 1995). These spectra show little or no variation over a wide range of timescales (Fenimore et al. 1994), both within individual bursts and between source active periods that cover years. However, there have been some exceptions in which a modest hard-to-soft evolution, within bursts from SGR 1806-20, was detected (Strohmayer \& Ibrahim 1998).

During the past 20 years, two giant flares have been recorded from two of the four known SGRs: one from SGR 0526-66 on 1979 March 5 (Mazets et al. 1979) and one from SGR 1900+14 on 1998 August 27 (Hurley et al. 1999a). These flares differ from the more common bursts in many ways. They are far more energetic (by 3 orders of magnitude in peak luminosity), persist for hundreds of seconds during which their emission is modulated at a period that reflects the spin of an underlying neutron star, and have harder initial spectra. The hard spectra of the peak of these flares have OTTB temperatures

\footnotetext{
${ }^{1}$ Department of Physics, University of Alabama in Huntsville, Huntsville, AL 35899; peter.woods@msfc.nasa.gov.

${ }^{2}$ NASA Marshall Space Flight Center, SD50, Huntsville, AL 35812.

${ }^{3}$ Universities Space Research Association.

${ }^{4}$ Astronomical Institute "Anton Pannekoek," University of Amsterdam, Kruislaan 403, Amsterdam, SJ, NL-1098, Netherlands.

${ }^{5}$ University of California, Berkeley, Space Sciences Laboratory, Berkeley, CA 94720-7450.

${ }^{6}$ Department of Physics and Astronomy, University of North Carolina, Phillips Hall, Chapel Hill, NC 27599-3255.

7 Department of Astronomy at Austin, University of Texas, RLM 15.308, Austin, TX 78712-1083.
}

of 200-500 keV (Fenimore, Klebesadel, \& Laros 1996; Hurley et al. 1999a; Mazets et al. 1999b), although this model should not necessarily be taken as an accurate description of these spectra since severe dead-time problems for most instruments limited the efficacy of spectral deconvolution. Feroci et al. (1999) require that the first $\sim 67$ s of the August 27 flare be fitted with a two-component spectrum, consisting of an OTTB $(31 \mathrm{keV})$ and a power law $(-1.47$ photon index $)$. Hard burst emission has also been detected during the brightest burst recorded from the newly discovered SGR 1627-41 (Woods et al. 1999c; Mazets et al. 1999a). Further evidence for hard emission from SGRs comes from Rossi X-Ray Timing Explorer (RXTE) observations of SGR 1806-20. For a small fraction of the more common short events, high OTTB spectral temperatures in the range of 50-170 keV were measured (Strohmayer \& Ibrahim 1998; Marsden 1999).

Here we present strong evidence for spectrally hard burst emission from SGR 1900+14 during its recent active episode that started in 1998 May. Two events recorded with the Burst and Transient Source Experiment (BATSE) that are positionally consistent with SGR 1900+14 and temporally coincident with the recent active period of the source show temporal and spectral signatures quite distinct from typical SGR burst emissions. We show that although the time-integrated spectrum of each event resembles a classical GRB spectrum, spectral evolution is found that obeys a hardness/intensity anticorrelation never before seen in GRBs.

\section{BURST ASSOCIATION WITH SGR $1900+14$}

On 1998 October 22, during a period of intense burst activity of SGR 1900+14 (Woods et al. 1999b), BATSE triggered at 15:40:47.4 UT on an $\sim 1$ s burst with a smooth, FRED-like (Fast Rise Exponential Decay) temporal profile. Such light curves are commonly seen in GRBs but are rare for SGR events, based on observations with BATSE and RXTE (Gögü̈ş et al. 1999). This burst was located near SGR 1900+14 (Fig. 1) but was longer than typical bursts from this source, 


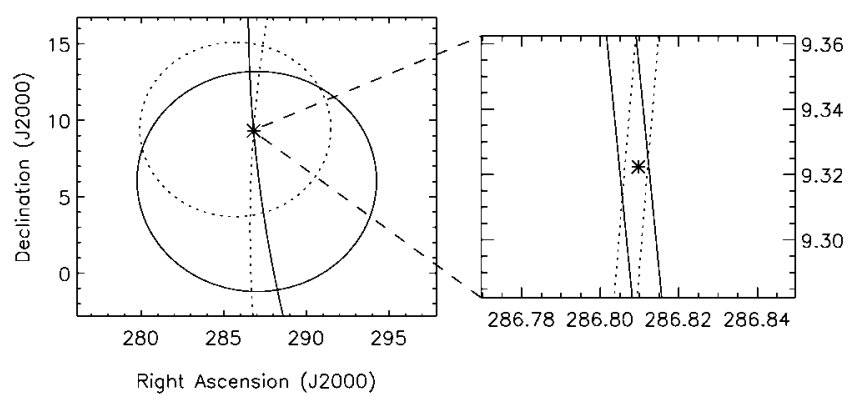

FIG. 1.-Localizations of 981022 (dotted) and 990110 (solid) with BATSE (circles denote $90 \%$ statistical + systematic error radii) and BATSE/Ulysses IPN arcs (99\% statistical + systematic error). The VLA location of SGR $1900+14$ is denoted by the asterisk.

and its spectrum was much harder ${ }^{8}$ (Fig. 2, top left panel). Using Ulysses and BATSE, an interplanetary network (IPN) annulus was constructed, and the joint BATSE/IPN error box (Fig. 1) contained the known source location of SGR 1900+14 (Frail, Kulkarni, \& Bloom 1999).

Without invoking any assumptions about source activity, we estimate the probability that a GRB within the BATSE database with an IPN arc would contain any known SGR by chance. For our purposes, we will assume that GRBs are isotropic and that the angular size of the known SGR error boxes are small compared with the joint BATSE/IPN error box. The probability $p$ reduces to $p \approx(1 / 4 \pi) N A$, where $N$ is the number of known SGRs and $A$ is the area of the burst error box in steradians. With four known SGRs, we find a chance probability of $3 \times 10^{-6}$ that a GRB with the given error box area will overlap a known SGR. We now normalize this probability by multiplying by the number of trials (i.e., the number of BATSE/ Ulysses IPN arcs as of 1999 March, which is 641), which gives the probability of a chance association of $2 \times 10^{-3}$. The burst was detected during a period when SGR $1900+14$ was active (a fact not used in the probability calculation), further strengthening the association.

Ten weeks later on 1999 January 10 at 08:39:01.4 UT, a strikingly similar burst (Fig. 2, top right panel) was recorded with BATSE, which again was located near SGR 1900+14. This burst also triggered Ulysses, and so an IPN annulus was constructed that again contained the position of SGR 1900+14 (Fig. 1). Using the same arguments as before, we find an upper limit to the probability that the burst and any known SGR are related by a chance coincidence of $3 \times 10^{-3}$. The combined probability (a product of the two individual probabilities) that these two events are GRBs with BATSE/IPN error boxes that are consistent with a known SGR by chance coincidence is $6 \times 10^{-6}$.

An alternative possibility is that these two bursts constitute two gravitationally lensed images of the same GRB (Paczyński 1986). However, we consider this highly unlikely given the positional coincidence of SGR $1900+14$, the temporal correlation with a known burst active period for the source, and the anticorrelation between hardness and intensity (see $\S 3$ ). We conclude that these two bursts originated from SGR 1900+14.

\section{BURST SPECTRA}

To fit the time-integrated spectrum for each burst, we used high-energy resolution burst data that have 128 energy channels

\footnotetext{
${ }^{8}$ The similarities of this event to GRBs in spectral and temporal structure was noted independently by Fargion (1999).
}

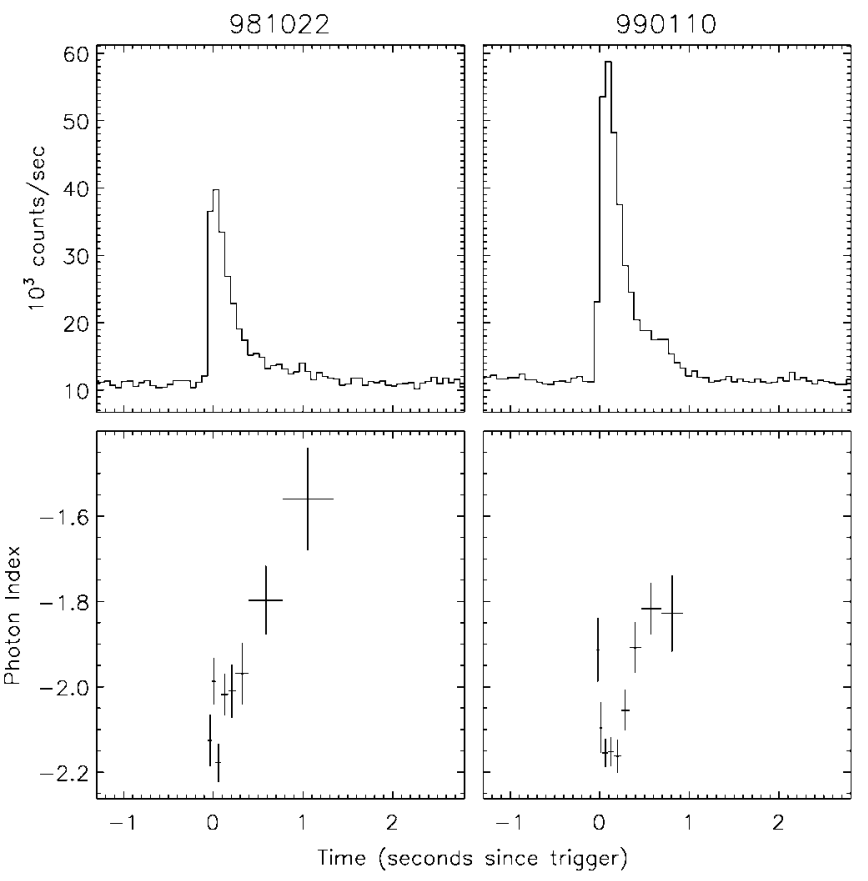

FIG. 2.-Light curves (25-2000 keV) of two bursts from SGR 1900+14 detected with BATSE. The BATSE trigger 7171 (981022) is shown in the top left panel, and trigger 7315 in the top right panel. The bottom panels give the photon index as a function of time to illustrate the spectral evolution observed during these events.

covering 20-2000 keV (see Fishman et al. 1989 for a description of BATSE data types). We fitted a third-order polynomial to approximately $300 \mathrm{~s}$ of preburst and postburst data and interpolated between these intervals to estimate the background at the time of the burst. This background was subtracted, and we fitted the resulting burst spectrum using WINGSPAN (WINdow Gamma SPectral ANalysis) to three models: an OTTB $\left[d N / d E \propto E^{-1} \exp (-E / k T)\right]$, a simple power law, and Band's GRB function (Band et al. 1993). We find that for each burst, the spectrum is not well characterized by the OTTB model based on the large value of $\chi_{v}^{2}$ (Table 1). Using a $\Delta \chi^{2}$ test between the Band and OTTB models (Band et al. 1997), we find that the Band function is strongly favored over the OTTB, with probabilities of $4.6 \times 10^{-7}$ and $7.2 \times 10^{-11}$ that these $\chi^{2}$ differences would occur by chance for the respective bursts. Furthermore, the Band function is favored over the simple power law for the second, brighter event with a significance level of $1.3 \times 10^{-3}$, although inclusion of a low-energy cutoff with the power-law model eliminates this advantage. Figure 3 shows the data and folded Band model for each burst.

For comparison with past results, we have included the OTTB fit parameters (Table 1) in order to demonstrate clearly

TABLE 1

Spectral Fit Summary

\begin{tabular}{ccrcc}
\hline \hline Burst & Model & \multicolumn{1}{c}{$\chi^{2} /$ dof } & $\begin{array}{c}k T \text { or } E_{\text {peak }} \\
(\mathrm{keV})\end{array}$ & Photon Index ${ }^{\mathrm{a}}$ \\
\hline $981022 \ldots \ldots$ & OTTB & $119.4 / 96$ & $102 \pm 5$ & $\ldots$ \\
& Power law & $92.7 / 96$ & $\ldots$ & $1.91 \pm 0.06$ \\
& Band's GRB & $90.2 / 94$ & $54 \pm 50$ & $1.96 \pm 0.08$ \\
OTTB & $139.8 / 95$ & $94 \pm 4$ & $\ldots$ \\
& Power law & $106.4 / 95$ & $\ldots$ & $2.06 \pm 0.03$ \\
& Band's GRB & $93.1 / 93$ & $59 \pm 11$ & $2.19 \pm 0.06$ \\
\hline
\end{tabular}

${ }^{a}$ For Band's GRB function, the high-energy index $\beta$ is given here. The low-energy index $\alpha$ is not well determined because of the low $E_{\text {peak }}$ values. 


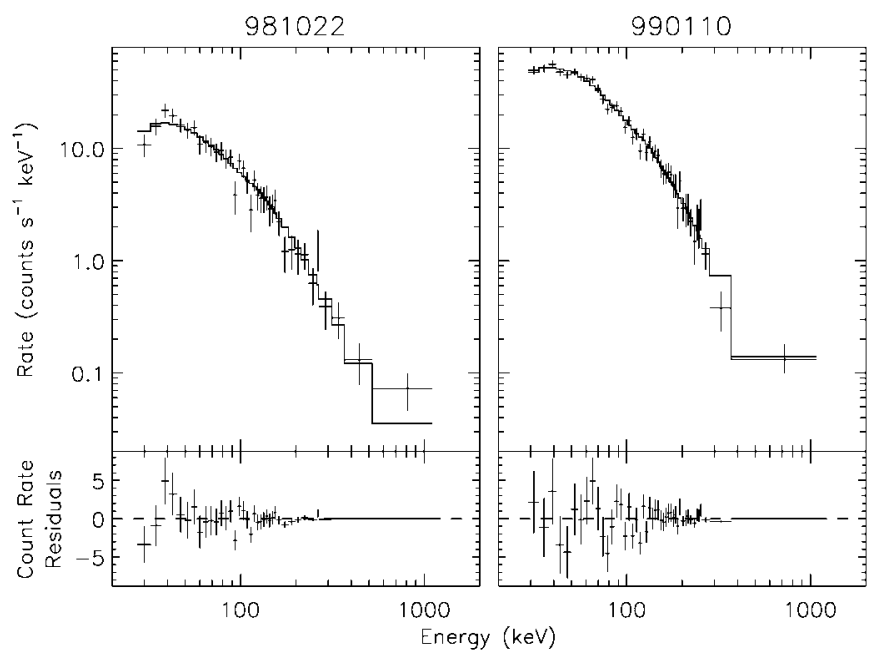

FIG. 3.-Count spectrum of 981022 (left) and 990110 (right) as fitted to Band's GRB function (see Table 1 for spectral fit information). The spectrum has been binned for display purposes.

the difference between the spectra of these bursts and typical SGR burst emissions. Specifically, for SGR 1900+14 during its recent active episode, a weighted mean of $25.7 \pm 0.8 \mathrm{keV}$ was found for the OTTB temperature of 22 events detected with BATSE (Göğüş et al. 1999). Clearly, the temperatures found for these two bursts are much higher than these typical values.

Using data with a coarser spectral resolution but a finer time resolution, we fitted multiple segments of each burst in order to search for the spectral evolution. For the initial rise of each event, we used time-tagged event data that have only four energy channels but a $2 \mu$ s time resolution. For the peak and tail, we used medium-energy resolution data that have 16 energy channels and a $16 \mathrm{~ms}$ time resolution. In order to sustain a good signal-to-noise ratio for a reasonable parameter determination, the bursts were divided into eight and nine intervals, respectively. Guided by our fit results of the time-integrated spectra and our limited number of energy channels, we chose to fit the power-law model to these spectra. We find significant spectral evolution through each burst as the power-law photon index varies between -1.5 and -2.4 (Fig. 2, bottom panels). We note a general soft-to-hard trend in the time evolution of the spectra of these bursts. This appears to be a consequence of a relatively faster temporal rise than decay and an intensity/ hardness anticorrelation for these events (Fig. 4). To quantify the significance of this correlation, we calculated the Spearman rank-order correlation coefficient $(\rho=-0.86)$ between the energy flux and the photon index. The probability of obtaining a coefficient of this value from a random data set is $8.3 \times$ $10^{-6}$; thus, the anticorrelation is significant.

The peak fluxes and fluences of the two events are not exceptional when compared with other burst emissions from this source. We find peak fluxes (0.064 s timescale) of $(2.94 \pm$ $0.15)$ and $(4.96 \pm 0.18) \times 10^{-6} \mathrm{ergs} \mathrm{cm}^{-2} \mathrm{~s}^{-1}$ and fluences $(25-2000 \mathrm{keV})$ of $(1.14 \pm 0.04)$ and $(1.85 \pm 0.04) \times 10^{-6}$ ergs $\mathrm{cm}^{-2}$ for the bursts of 981022 and 990110 , respectively. For a distance of $7 \mathrm{kpc}$ (Vasisht et al. 1994), these correspond to peak luminosities of 1.7 and $2.9 \times 10^{40} \mathrm{ergs} \mathrm{s}^{-1}$ and burst energies of 0.65 and $1.1 \times 10^{40}$ ergs (assuming isotropic emission). The ranges of peak fluxes and fluences for SGR $1900+14$ bursts observed recently with BATSE are (0.3-20) $\times 10^{-6}$ and $(0.02-25) \times 10^{-6} \mathrm{ergs} \mathrm{cm}^{-2}$, respectively (Gögüus et al. 1999).

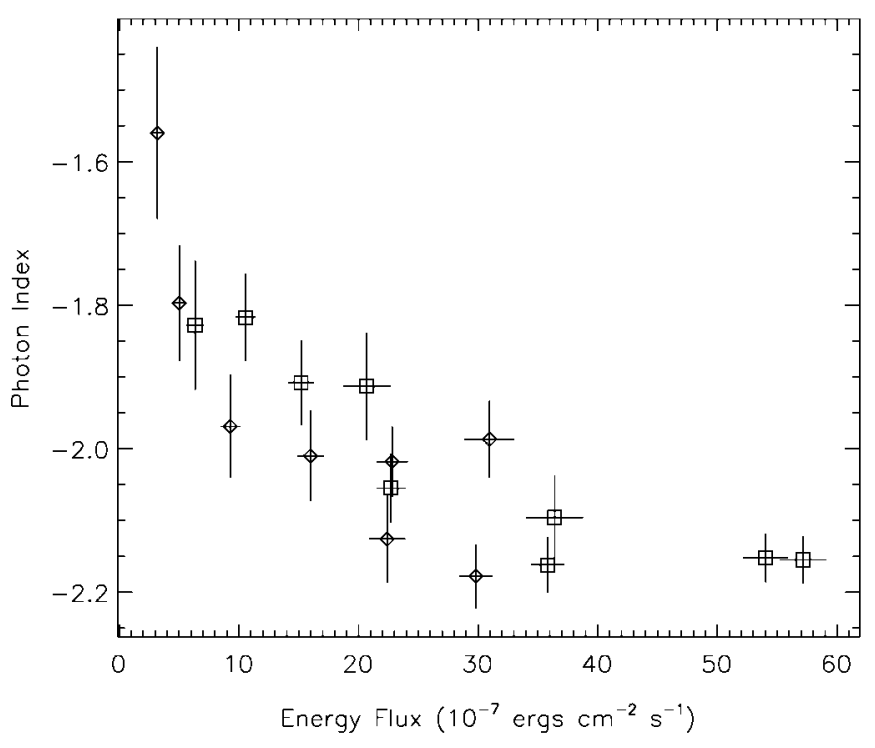

Fig. 4.-Energy flux vs. photon index for 981022 (diamonds) and 990110 (squares).

The measured values for these bursts with harder spectra are well within the corresponding observed ranges.

\section{A COMPARISON WITH OBSERVED GRB CHARACTERISTICS}

For each event, we have calculated two quantities that are traditionally used to delineate between the two classes (Kouveliotou et al. 1993) of GRBs in the BATSE catalog, specifically $T_{90}$ and spectral hardness (100-300 keV/50-100 keV). We find $T_{90}$ durations of $1.2 \pm 0.2$ and $0.9 \pm 0.2 \mathrm{~s}$ and fluence hardness ratios of $1.9 \pm 0.1$ and $2.1 \pm 0.1$ for the bursts of 981022 and 990110, respectively. When plotted together with the reported values of the $4 \mathrm{Br}$ catalog (Paciesas et al. 1999), we find that these two bursts fall outside the main concentrations of each distribution (i.e., the long, soft class and the short, hard class) but nearer the centroid of the short, hard class. We are currently looking more closely at events in the same region of this diagram that were classified as GRBs.

Given the spectral similarities between these two events and a fair fraction of GRBs, the time-integrated spectrum is not sufficient to distinguish these bursts from GRBs. Many GRBs show a strong hardness/intensity correlation within individual bursts (Ford et al. 1995; Preece et al. 1999), but to the best of our knowledge, a consistent anticorrelation throughout a GRB has never been seen. The two bursts from SGR 1900+14, however, do show a strong hardness/intensity anticorrelation (Fig. 4). If this behavior is inherent to all hard SGR events, it would be a useful diagnostic (but secondary to location) with which to select them.

\section{DISCUSSION}

We have shown strong evidence for hard emission from SGR $1900+14$ during two bursts of average intensity as observed with BATSE. It is clear that this type of burst emission in SGR $1900+14$ is rare $(1 \%$ of the SGR $1900+14$ events acquired with BATSE during the 1998-1999 active period). Their occurrence following the August 27 flare may suggest a causal relationship, but this is difficult to pin down given the rarity of hard events and the enhanced burst activity at the time. The clear distinction between these two events in hardness, spectral form, spectral evolution, duration, and lack of temporal vari- 
ability suggests these bursts are created either by physical processes different from those that produce the more common, soft events or in a region whose ambient properties that affect the emitted radiation (e.g., magnetic field, optical depth, etc.) are different.

The proposed identification of SGRs with very strongly magnetized neutron stars (Duncan \& Thompson 1992) has received strong support from the discovery that both SGR 1806-20 and SGR $1900+14$ are X-ray pulsars spinning down at rapid rates (Kouveliotou et al. 1998, 1999; Hurley et al. 1999b). In this magnetar model, the typically soft SGR bursts are explained in the following way. The internal magnetic field is strong enough to diffuse rapidly through the core, thereby stressing the crust (Thompson \& Duncan 1996). A sudden fracture injects a pulse of Alfvén radiation directly into the magnetosphere, which cascades to a high wavenumber and creates a trapped fireball (Thompson \& Duncan 1995; Thompson \& Blaes 1998). The soft spectrum arises from a combination of photon splitting and Compton scattering in the cool, matterloaded envelope of the fireball.

The relative hardness of the two reported events, combined with luminosities in excess of $10^{40} \mathrm{ergs}^{-1}$, points directly to an emission region of shallower scattering depth, $\tau_{\mathrm{es}}<1$, situated outside $\sim 10^{3}\left(L_{\mathrm{X}} / 10^{40} \mathrm{ergs} \mathrm{s}^{-1}\right)$ neutron star radii. Inverse Compton emission is suggested by the lack of a correspondence between the spectral break energy and a cooling energy at this large a radius. For example, bulk Alfvén motions are an effective source of Compton heating even in the absence of Coulomb coupling between electrons and ions (Thompson 1994). In certain circumstances, one expects that Alfvén radiation will disperse rapidly throughout the magnetosphere, if the initial impulse occurs on extended dipole field lines or if it involves a buried fracture of the crust. This rapid dispersal is made possible by the strong coupling between external Alfvén modes and internal seismic waves (cf. Blaes et al. 1989). The wave energy is then distributed logarithmically with radius, with the wave amplitude $\delta B / B$ approaching unity at the Alfvén radius $R_{\mathrm{A}} / R_{\star} \simeq 900\left(B_{\star} / 4.4 \times 10^{14} \mathrm{G}\right)^{1 / 2}\left(L_{\mathrm{A}} / 10^{40} \mathrm{ergs} \mathrm{s}^{-1}\right)^{-1 / 4}$ (Thompson \& Blaes 1998). Here $B_{\star}$ is the polar dipole magnetic field, $L_{\mathrm{A}}$ is the luminosity in escaping waves, and $R_{\star} \simeq 10 \mathrm{~km}$. Damping by leakage onto open field lines (which extend beyond the Alfvén radius) occurs on a timescale of $t_{\text {damp }}=3\left(2 R_{\mathrm{A}}\right.$ ' $\left.R_{\star}\right)\left(R_{\star} / c\right)=0.2\left(B_{\star} / 4.4 \times 10^{14} \mathrm{G}\right)^{1 / 2}\left(L_{\mathrm{A}} / 10^{40} \mathrm{ergs} \mathrm{s}^{-1}\right)^{-1 / 4} \mathrm{~s}$. This lies close to the FWHM of the two reported bursts. Wave excitations near the neutron star undergo a turbulent cascade on a similar timescale and will generate softer seed X-ray photons (C. Thompson et al. 1999, in preparation).

Independent of the detailed physical mechanism, these observations demonstrate that a Galactic source-probably a strongly magnetized neutron star with a large velocity-is capable of producing a burst of gamma rays whose timeintegrated spectrum resembles that of a classical GRB. The similarity is remarkable in light of the difference in peak luminosities of $\gtrsim 10^{11}$ (modulo beaming factors). However, the two bursts from SGR $1900+14$ presented here are by no means "typical" GRBs, given their low $E_{\text {peak }}$ values, unusual spectral evolution, and their position in the duration-hardness plane. Furthermore, we know that the currently active SGRs in our Galaxy lie close to the Galactic plane $\left(z_{\mathrm{rms}} \simeq 66 \mathrm{pc}\right)$, so their contribution to the BATSE GRB catalog must be minimal on account of the isotropic spatial distribution of GRBs. A larger contribution from older magnetars that have moved away from the Galactic plane is conceivable, but it would require that the preponderance of hard bursts to soft bursts increases tremendously with age.

We thank Chip Meegan for useful discussions. P. M. W. acknowledges support under the cooperative agreement NCC 8-65. J. v. P. acknowledges support under NASA grants NAG53674 and NAG5-7060. K. H. is grateful for support under JPL contract 958056 and NASA grant NAG5-7810. C. T. acknowledges support from the Alfred P. Sloan Foundation.

\section{REFERENCES}

Band, D. L., Ford, L. A., Matteson, J. L., Briggs, M. S., Paciesas, W. S., Pendleton, G. N., \& Preece, R. D. 1997, ApJ, 485, 747

Band, D., et al. 1993, ApJ, 413, 281

Blaes, O., Blandford, R., Goldreich, P., \& Madau, P. 1989, ApJ, 343, 839

Duncan, R., \& Thompson, C. 1992, ApJ, 392, L9

Fargion, D. 1999, preprint (astro-ph/9903433)

Fenimore, E., Klebesadel, R., \& Laros, J. 1996, ApJ, 460, 964

Fenimore, E., Laros, J., \& Ulmer, A. 1994, ApJ, 432, 742

Feroci, M., Frontera, F., Costa, E., Amati, L., Tavani, M., Rapisarda, M., \& Orlandini, M. 1999, ApJ, 515, L9

Fishman, G. J., et al. 1989, in Compton Observatory Science Workshop, ed. W. N. Johnson (Greenbelt: NASA/GSFC), 2

Ford, L. A., et al. 1995, ApJ, 439, 307

Frail, D., Kulkarni, S., \& Bloom, J. 1999, Nature, 398, 127

Göğüş, E., Woods, P. M., Kouveliotou, C., van Paradijs, J., Briggs, M. S., Duncan, R. C., \& Thompson, C. 1999, ApJ, in press

Hurley, K., et al. 1999a, Nature, 397, 41

1999b, ApJ, 510, L111

Kouveliotou, C. 1995, Ap\&SS, 231, 49

Kouveliotou, C., Meegan, C. A., Fishman, G. J., Bhat, N. P., Briggs, M. S.,

Koshut, T. M., Paciesas, W. S., \& Pendleton, G. N. 1993, ApJ, 413, L101

Kouveliotou, C., et al. 1998, Nature, 393, 235

- 1999, ApJ, 510, L115

Marsden, D. 1999, Ph.D. thesis, Univ. California, San Diego
Mazets, E. P., Aptekar, R. L., Butterworth, P., Cline, T., Frederiks, D. D., Golenetskii, S. V., Hurley, K., \& Il'inskii, V. N. 1999a, ApJ, 519, L151

Mazets, E. P., Cline, T., Aptekar, R. L., Butterworth, P., Frederiks, D. D., Golenetskii, S. V., Il'inskii, V. N., \& Pal'shin, V. D. 1999b, Astron. Lett., submitted

Mazets, E. P., Golenetskii, S. V., Il'inskii, V. N., Aptekar, R. L., \& Guryan, Yu. A. 1979, Nature, 282, 587

Paciesas, W., et al. 1999, ApJS, 122, 465

Paczyński, B. 1986, ApJ, 308, L43

Preece, R. D., Briggs, M. S., Mallozzi, R. S., Pendleton, G. N., Paciesas, W. S., \& Band, D. L. 1999, ApJS, in press

Strohmayer, T., \& Ibrahim, A. 1998, in AIP Conf. Proc. 428, Fourth Huntsville Symp. on Gamma-Ray Bursts, ed. C. A. Meegan, R. D. Preece, \& T. M. Koshut (Woodbury: AIP), 947

Thompson, C. 1994, MNRAS, 270, 480

Thompson, C., \& Blaes, O. 1998, Phys. Rev. D, 57, 3219

Thompson, C., \& Duncan, R. 1995, MNRAS, 275, 255

. 1996, ApJ, 473, 322

Vasisht, G., Kulkarni, S., Frail, D., \& Greiner, J. 1994, ApJ, 431, L35

Woods, P., Kouveliotou, C., van Paradijs, J., Finger, M. H., \& Thompson, C. 1999a, ApJ, 518, L103

Woods, P., et al. 1999b, ApJ, 524, L55 . 1999c, ApJ, 519, L139 